\title{
A Multiclass, Multiproduct Covid-19 Convalescent Plasma Donor Equilibrium Model
}

\author{
Anna Nagurney ${ }^{1} \cdot$ Pritha Dutta ${ }^{2}$ D
}

Received: 25 May 2020 / Accepted: 27 May 2021/ Published online: 10 July 2021

(c) The Author(s), under exclusive licence to Springer Nature Switzerland AG 2021

\begin{abstract}
In this paper, we develop a multiclass, multiproduct equilibrium model for convalescent plasma donations in the Covid-19 pandemic. The potential donors are situated at different locations and the donor population at each location can be separated into different classes based on their motivation and the product for which they provide donations at a collection site. The model captures the competition between nonprofit and for-profit organizations seeking convalescent plasma donations, which is a characteristic of this new market. A variational inequality formulation of the equilibrium conditions and qualitative properties of the model are provided. We also present a capacitated version of the model. Numerical examples of increasing complexity are presented and solved using the modified projection method. The results reveal multiclass, multiproduct donor behavior under different scenarios which can inform policy makers during this pandemic and beyond.
\end{abstract}

Keywords Convalescent plasma $\cdot$ Pandemic $\cdot$ Covid-19 $\cdot$ Blood donations ·

Networks

\section{Introduction}

In the first quarter of 2020, the Covid-19 pandemic disrupted the globe, severely compromising economic, educational, and social activities as well as healthcare. Although the vaccination process has been ongoing steadily since the beginning of 2021 in the USA and several other countries across the world, we are a long way from fully vaccinating the entire world's population. Thirty-eight percent of the population in the USA, 40 percent of the population in Israel, and 32 percent of the population in UK are fully

Pritha Dutta

pdutta@pace.edu

1 Department of Operations and Information Management, Isenberg School of Management, University of Massachusetts, Amherst 01003, Massachusetts, USA

2 Department of Management and Management Science, Lubin School of Business, Pace University, 10038 New York, USA 
vaccinated, as of May 2021. However, for many countries this number is still in the $20 \mathrm{~s}$ or even lower. For a complete list of countries and vaccination rates, please refer [1]. The economic losses, pain, suffering, and the number of deaths, on the other hand, still continue to rise with new cases, the discovery of new variants in various countries such as India, UK, Brazil [2, 3], and the catastrophic impact of the second wave in India [4].

\subsection{Covid-19 and Convalescent Plasma Therapy}

One treatment that has been implemented in this time of crisis, especially when and where vaccines were not available, is that of convalescent plasma therapy [5-8]. Such convalescent plasma is the plasma or liquid part of blood obtained from recovered Covid-19 patients which contains antibodies that can fight the virus SARSCoV-2 causing the disease [9]. The treatment gained prominence in the fight against coronavirus when studies including one on 39 Covid-19 patients by researchers at Mount Sinai Medical Center in New York found a trend towards better survival rates for patients who received convalescent plasma [10]. Following these research findings in 2020 the US Food and Drug Administration allowed the use of convalescent plasma during the pandemic because there was no approved treatment for Covid19. As more Covid-19 patients recovered from the coronavirus, which originated in Wuhan, China, the supply of convalescent plasma from willing donors increased.

While there is consensus that there is no adverse effect, the extent of the impact of convalescent plasma therapy on the recovery of Covid-19 patients is inconclusive and complicated, depending on factors such as the severity of infection, amount of convalescent plasma transfused and the antibody status and titers in donors and recipients $[11,12]$. Investigations in clinical trials are still going on [13] and some recent studies have even found no significant benefits of the treatment on patients [14]. Many of these trials focused on severely ill hospitalized patients despite findings and theoretical considerations supporting administration of convalescent plasma at the onset of infection rather than at a later stage [12]. Convalescent plasma therapy has also been used to treat other infectious diseases in the past [15]. In [16] the authors reported findings from a study on 80 patients who were given convalescent plasma in Hong Kong during the 2003 SARS outbreak. A higher discharge rate at day 22 was observed among patients who received convalescent plasma before day 14 of illness and among those who were PCR positive and seronegative for coronavirus at the time of plasma transfusion. However, this was not a randomized trial and the antibody titers and plasma transfusion volumes varied but did not appear to correlate with clinical response. Several studies were carried out to test the effectiveness of convalescent plasma treatment in the case of the Ebola virus infection [17, 18]. Mair-Jenkins et al. [19] conducted an exploratory meta-analysis that showed the positive effects of using convalescent plasma for the treatment of severe acute viral respiratory infections, including those caused by related coronaviruses (SARS-CoV and MERS-CoV).

Convalescent plasma therapy received attention globally for Covid-19, as governments desperately looked for any possible treatments and clinical trials for this investigational therapy got approved in several parts of the world. The European 
Commission, jointly with the European Blood Alliance (EBA), the European Center for Disease Prevention and Control (ECDC), and healthcare professionals is driving the study on convalescent plasma treatment in Europe. EBA is emphasizing data reporting and information sharing among blood banks and clinicians through an open database that they are developing to ensure immediate availability of critical information on donor selection, plasma collection processes, etc. [20]. At present, several studies are being conducted focusing on early transfusion of high potency convalescent plasma supported by European Union research funding [21]. At the beginning of May 2020, the National Ethics Committee in India also approved the Indian Council of Medical Research's (ICMR) request to conduct clinical trials in 21 hospitals across the country to study the effectiveness of convalescent plasma treatment [22]. As India's healthcare system struggled to manage the disastrous impact of the second wave of the pandemic, relatives of patients and even government agencies such as Delhi Police put out desperate calls for convalescent plasma donations in large numbers [23]. However, on May 17, 2021, the Indian Health Ministry and AIIMS-ICMR Covid-19 National Task Force withdrew convalesecnt plasma therapy from their Covid-19 treatment protocol in light of recent findings that do not show any therapeutic benefits of the treatment on Covid-19 patients [14].

In 2020, the US Food and Drug Administration (FDA), in partnership with academics and industry, began a nation-wide effort to facilitate the development of two investigational therapies: one on convalescent plasma treatment and the other on hyperimmune globulin [24]. Hyperimmune globulin is a concentrated antibody serum derived from plasma that can possibly prevent or terminate infection in the future [25]. There exists a possible third treatment, monoclonal antibody therapy. This therapy uses antibody-producing cells from high-antibody donors to produce laboratory-produced molecules to battle Covid-19. According to some scholars, until herd immunity is attained, either through mass vaccination and/or natural infection, these products have the potential to provide passive antibody-based immunity to individuals who were previously unexposed to reduce the risk of disease or to reduce its clinical impact in case an individual is already infected [26].

In the USA, researchers, physicians, nonprofit organization such as blood banks, hospitals, the Mayo Clinic, and government regulatory bodies came together to investigate the merits of this therapy. In order to drive collections, they tried to raise awareness about convalescent plasma treatment among recovered individuals through announcements on their websites and initiatives such as the National Covid-19 Convalescent Plasma Project. In April 2020, the FDA launched a website to guide recovered Covid-19 patients to plasma collection centers and issued guidelines for plasma collection by nonprofit blood banks such as the American Red Cross, the New York Blood Center, as well as hospitals, for treating severely ill patients [27]. The American Red Cross started collecting convalescent plasma at over 170 locations across the country [28]. On August 23, 2020, the FDA issued an emergency use authorization (EUA) for the use of convalescent plasma in treating hospitalized Covid-19 patients, which was reissued on December 1 [29]. As several areas across the USA experienced an increase in the number of Covid-19 cases, blood banks continued to recruit and call for recovered patients to donate plasma 
$[30,31]$. Blood banks such as the MEDIC blood bank in East Tennessee expanded convalescent plasma collection at its donor centers and added mobile centers in order to meet the surge in demand [32]. In the USA, over 250,000 units of convalescent plasma have been administered either as part of EUA or through a clinical trial [26]. However, in January and February of 2021, the FDA revised the terms and conditions of the EUA, cutting back on the use of convalescent plasma therapy as the number of infections and, subsequently, demand decreased steadily in the country [33]. It is evident the market for convalescent plasma has been rapidly evolving over the past year with changes in the severity of the pandemic and emerging results on the efficacy of the therapy from various clinical trials. Nevertheless, it is important to have an inventory for use in case of future outbreaks or subsequent waves of the Covid-19 pandemic.

\subsection{Convalescent Plasma Donation}

According to FDA's guidelines [34], individuals who have fully recovered from Covid-19 and have shown no symptoms for at least 2 weeks prior to donation are eligible to donate plasma. The donors must have had a prior diagnosis of Covid-19 documented by a laboratory test. Those who did not have a prior positive diagnostic test and/or never had symptoms of COVID-19 may also be eligible to donate if they have had positive results in two different tests approved, cleared, or authorized by FDA to detect SARS-CoV-2 antibodies. In addition, all donors must meet the regular donor criteria of age and weight and have passed a medical history screening in order to maintain public health safety. The time of donation is also crucial. In [35] the authors identified the optimal time window for donation before virusneutralizing antibodies start decreasing in recovered Covid-19 patients and important characteristics of ideal donors such as antibody titers. As of May 2020, eligible donors could give convalescent plasma to blood banks once every 28 days. Since August 31, 2020, the American Red Cross has been accepting donations from individuals once every seven days for up to three months, but a maximum of eight times [36]. Nevertheless, the donor pool for convalescent plasma is limited. The plasma collection process involves drawing blood from one arm of the donor and sending it through a high-tech machine. The machine collects the plasma separating it from whole blood and then safely returns the red cells back to the donor. According to the Blood Bank of Delmarva the apheresis process takes 45-50 minutes. However, the total visit time including medical screening is over at around 75 minutes. After collection, similar to Fresh Frozen Plasma (FFP), convalescent plasma should be frozen within 8 hours and stored at $-18 \mathrm{C}$ or colder. The manufacturing process for Covid-19 convalescent plasma also includes putting the expiration date of one year from the date of collection on the label [34]. However, once thawed, plasma must be transfused within 5 days.

While nonprofit blood banks and hospital blood programs have been seeking convalescent plasma for clinical trials conducted by research groups and for treatment of severely ill patients, profit-making companies also started collecting this potentially life-saving product for supplying blood samples to laboratories and test 
manufacturers with monetary compensations [37, 38]. Profit-making companies typically offer $\$ 50$ to donors for Covid-19 plasma donations. However, the Takeda Pharmaceuticals' BioLife Plasma Services Center, which is part of an industry producing plasma protein therapies crucial for treating certain rare chronic conditions, has reportedly provided gift cards worth $\$ 800$ to a couple for making two donations [25]. In times of crisis such as this, it is natural for many people to act altruistically to help the community. For example, in the aftermath of $9 / 11$, there was a surge of blood donations, especially from first time donors which eventually led to wastage of collected blood [39]. However, in the current pandemic scenario, donors may not only derive satisfaction or utility from their altruistic behavior but also receive monetary benefits if they choose to donate to a profit-making company such as Cantor BioConnect, which sells the plasma at high prices to laboratories and test manufacturers even overseas [37].

It is evident from the above discussion that an interesting market for convalescent plasma, which can be used to treat patients and to develop products such as hyperimmune globulin and other critical therapies, has emerged. This market involves multiple players with different objectives and a limited donor pool. Donors, hence, have several options for donation in terms of the product and the organization. In some cases, individuals who are not eligible to donate plasma for direct treatment due to safety concerns, such as Hasidic women who have recovered from Covid-19, can still choose to donate for hyperimmune globulin creation [25]. Donors are able to distinguish among the competing organizations collecting convalescent plasma based on their purpose and also the product and its use as well as their own conditions to meet the eligibility criteria for donation for a particular product.

Several studies across different disciplines, including economics, psychology, medicine, and operations research [40-44], have been conducted in order to better understand the motivation behind blood donations. In their paper, Evans and Ferguson [42] reported on a psychometric assessment of altruism that can inform and guide donor recruitment strategies. They used five theoretical dimensions of altruism; namely, impure altruism, kinship, self-regarding motives, reluctant altruism, and egalitarian warm glow to differentiate between donors and non-donors. Their findings suggest that pure altruism is often not the sole motivation for voluntary blood donors, and that blood donor behavior is rather complex. Based on this analysis, we can assume that the blood or, in this case, the convalescent plasma donor population is not homogeneous and can be separated into several classes on the basis of their motivation as well as appropriate fit for a specific product.

\subsection{Contributions}

Convalescent plasma has proved to be a crucial component in the healthcare system during a global pandemic in the absence of vaccine and approved treatments. On the other hand, the market for convalescent plasma in the for-profit sector is expected to grow from 0.026 billion USD in 2020 to 0.028 billion USD in 2021 at a compound annual growth rate (CAGR) of 7.7 percent according to a report by The Business Research Company published in March 2021. Concerned by the 
growing competition between the nonprofit and for-profit organizations Farrugia et al. in [45] wrote "We apprehend that potential CP (convalescent plasma) donors who may approach the community blood sector for altruistic reasons may be deflected to the commercial sector through the high remuneration offered. This may be accentuated during this period as the traditionally low-resource population of paid plasma donors may be further augmented through the difficult economic situation, as occurred in previous economic crises". Hence, it is important to study the behavior of donors in this competitive scenario that can also help government agencies prepare for future waves of the Covid-19 pandemic or other infectious disease outbreaks. In this paper, we develop a novel multiclass, multiproduct donor model for convalescent plasma donations. To the best of our knowledge, this is the first model to capture competition between nonprofit organizations and for-profit organizations for the collection of convalescent plasma. The model is an equilibrium model and consists of different classes of recovered Covid-19 survivors who meet the criteria for donating convalescent plasma for one or more of the plasma products. The donors are located at origin locations whereas the plasma collection sites are located at destination locations. Each class of donors at an origin location has an associated utility function of donating the plasma for a particular purpose/product at a collection site. There is also a generalized cost, which includes, time, money, potential risk, etc., of going from the origin location to each collection site for a class of donor at an origin. We assume a fixed population of recovered donors of each class at each origin location. The donors reflect their preferences through their utility functions.

We now provide a discussion of the existing literature of relevance to our contributions in this paper. We do emphasize that the topic under consideration in this paper is especially timely and novel as the events surrounding the Covid-19 pandemic rapidly evolve. Furthermore, the unusual market for convalescent plasma with both nonprofits and for-profit organizations involved in competing for the plasma in the blood of recovered is quite special and merits investigation.

\section{Literature Review}

\subsection{Charitable Donations and Donor Behavior}

We begin the discussion in this section by highlighting existant literature on the modeling of charitable donations, donor behavior, and preferences. Toyasaki and Wakolbinger [46] compared two common fundraising techniques using optimization models: fundraising with the option of earmarking donations and fundraising without an earmarking option. They modeled the donor's behavior as well as the relief agency's behavior to analyze the effect of earmarking donations on fundraising costs. Saxton and Zhuang [47] proposed a game theory model that showed that the amount of charitable contributions made by donors is positively dependent on the amount of disclosure by the nongovernmental organizations (NGOs). The authors distinguish between two core donor preferences: the desire for impact and the desire for publicity, with donors choosing between organizations based on how well the organization satisfies these preferences. Zhuang et al. [48] further showed that the 
disclosure of financial performance, donor relations, and fundraising information is an important tool for nonprofit organizations to attract greater donations as it boosts accountability and public trust. Game theory has also been used to investigate other types of charitable donations such as organ donations [49].

\subsection{Human Migration Models}

There exists a rich body of literature on equilibrium models with varied applications and using the formalism of variational inequalities (cf. Kinderleher and Stampacchia [50]), originating in such classical contributions as those of [51, 52] on traffic network equilibrium; that of [53] on oligopolistic market equilibria, as well as that of [54] on spatial price equilibria. For recent contributions and applications, see the edited volumes by [55-58], and the references therein. In this paper, we focus specifically on equilibrium models developed for humanitarian and public policy issues that have significant societal impact (cf. Muggy and Stamm [59] and the references therein). Our modeling framework is related to that being utilized in models of human migration. For example, $[60,61]$ studied human migration with multiclass equilibrium models. Nagurney et al. [62, 63], subsequently, expanded the work in this area and developed multiclass human migration models with movement/migration costs and class transformation. Causa, Jadamba, and Raciti [64] also built on the model of [61] to incorporate uncertainty in the utility functions, the migration cost functions, and the populations. More recently, [65] developed a network model with regulations for international human migration problems. Their analysis provides insights on the effects of regulations on the utilities of different classes of migrants.

However, although the models in this paper make use of utility functions and generalized costs associated with origin and destination pairs, as has been done in various migration network models with migration costs (see also Nagurney [66]), here, we have added features uniquely relevant to convalescent plasma donations in the pandemic. These features include the purpose/product of the donation at each collection site plus the capacities at the sites. Furthermore, in this paper, the collection sites can be nonprofit or profit ones, which has no analogue in migration models.

\subsection{Blood Supply Chains}

In the domain of the blood supply chain literature, of specific relevance to this paper, is the work of [67]. That paper developed a multitiered integrated blood supply chain competition model with different stakeholders, namely blood banks, hospitals, and patient payer groups such as Medicare, Medicaid, private insurance companies, etc. The model is based on a blood product pricing scheme aimed at ensuring the economic sustainability of the blood banking industry which is essential for maintaining a steady supply of safe blood in the country. The results of their analysis reveal the benefit of a cost-based pricing scheme for blood products that takes into account the volume of blood transfused and the actual costs of all the supply chain operations can inform policymakers. 
While financial stress on blood banks due to increased competition, reduced demand, and cost-intensive testing operations has been a concern in the blood banking industry in recent times, the challenges associated with blood supply chain management are vast and varied. For a broad literature review on this topic see Nagurney and Dutta [44] and Dutta and Nagurney [67]. In this paper, we mention a few recent works on this topic. Piraban et al. [68] provide an up-to-date literature review on blood supply chain management. Osorio et al. [69] use discreteevent simulation to capture flows through the blood supply chain consisting of collection, production, storing, and distribution and an integer linear optimization model running over a rolling horizon to decide the required number of donors, collection methods, and production planning. As the authors point out in their paper, blood supply chain research often deals with specific stages of the supply process. There are few works that integrate all the operations comprising the supply chain (cf. Masoumi et al. [70]). Given the life-saving properties of blood products there has been a growing emphasis on research focusing on designing robust blood supply chains in the case of emergencies and disasters [71-74].

Although the above mentioned studies tackle interesting and significant issues, there is a clear dearth of modeling research on blood donor behavior which is a critical and unique part of supply chain management of this life-saving product, which cannot be produced but must be donated. Hosseini-Motlagh et al. [75] developed a novel mixed possibilistic-stochastic flexible robust model that takes into consideration the critical role played by blood donors. Their study included motivational initiatives aimed at encouraging blood donors in order to maintain a sufficient blood supply. The authors incorporate factors such as advertisement, education, and medical credits in constructing the motivational function.

There has been extensive research on blood donor recruitment and retention drawing from theories in social and behavioral sciences [76]. Much of that research is empirical work $[41,77,78]$. While the theoretical framework of blood donation focuses on altruism [40] as the driving force behind blood donations, there have been studies that investigate other potential sources of motivation. Lacetera et al. [79] discussed the effect of rewards and incentives on blood donations to mitigate shortages. In [80] the authors proposed two incentive schemes to drive convalescent plasma donation where under the first scheme, donors obtain treatment vouchers that can be transferred to patients of their choosing and under the latter scheme, patients obtain priority for convalescent plasma therapy in exchange for a future pledge to donate plasma if possible. Analytical results in the paper show that in steady state, both principles can increase overall treatment rates for all patients in need of convalescent plasma therapy. Nagurney and Dutta [44] modeled the competition among blood banks for donations from voluntary donors using the level of service quality provided at the blood collection sites as factors driving donations. In this paper, we advance the work on the modeling of donor behavior, specifically, in the case of convalescent plasma in relation to the Covid-19 pandemic, which has unique features, including nonprofit as well as forprofit organizations collecting the plasma. 


\subsection{Covid-19}

We would also like to mention research pertaining to the Covid-19 pandemic. Kabir and Tanimoto [81] use the framework of evolutionary game theory to model behavioral dynamics associated with economic shutdowns implemented by governments in response to the spread of Covid-19. They obtain valuable insights that can help governments in decision making, one of them being that "shield immunity takes hold in a population most easily when a lockdown is enacted with relatively low costs to the individuals." Kaplan [82] presents probability models that are used to assess the effectiveness of two policies adopted by countries to contain the outbreak of the infectious disease; namely, case isolation and quarantine within a community during the initial phase of outbreak. Baveja et al. [83] propose a plan with four strategies to be implemented over a period of 90 days in order to stop the spread of the infectious disease while mitigating its economic repercussions: (a) stop all international, domestic passenger air and intercity bus/ train travel; (b) create administrative zones of about 1 million people; (c) stop all non-emergency cross-zonal travel except for transportation of goods, and (d) deploy an information-driven service value chain to control the spread of the pandemic within a zone. In another recent publication, Ivanov [84] develops a new concept of Viable Supply Chain (VSC) drawing from the principles of agility, resilience, and sustainability. The author mentions that the VSC model can help firms make decisions on recovery and re-building of their supply chains in the aftermath of a global crisis such as the Covid-19 pandemic. Rassia [85] presents an interesting perspective on the need to focus on human values and human interaction with nature in case of architectural designs to create safe spaces protecting people in conditions of crisis. The Covid-19 outbreak is a good example to study since the best way to avoid the infection is to stay indoors; specifically, at home. Nagurney [86], in turn, constructs a game theory supply chain network model, which includes labor as a critical resource, and which includes labor productivity associated with supply chain economic activities. The framework considers three different sets of constraints on labor availability, and is inspired by the Covid19 pandemic. The author also presents numerical examples associated with pandemic labor disruptions. Choi [87] discusses how the powerful methodologies of operations research can "come to the rescue" in the pandemic and classifies the literature into before the pandemic, during the pandemic, and after the pandemic. $\mathrm{He}$ also highlights what specific actions governments, healthcare, various nonprofit-making organizations, and businesses should take to deal with Covid-19.

The remainder of the paper is organized as follows. In Section 3, we construct the multiclass, multiproduct convalescent plasma donor model, state the equilibrium conditions, and provide the variational inequality formulation. We also present an extension that includes capacity-like constraints. In Section 4 , we provide qualitative properties of the model, along with an algorithm. In Section 5, we apply the algorithm to compute solutions to numerical examples in order to illustrate the modeling and algorithmic framework and the types of insights that can be gained. Section 6 summarizes the results and present our conclusions, along with suggestions for future research. 


\section{The Multiclass, Multiproduct Convalescent Plasma Donor Equilibrium Model}

\subsection{The Uncapacitated Equilibrium Model}

The model consists of $m$ locations at which recovered Covid-19 individuals are located with a typical such origin location denoted by $i$. There are also $n$ locations at which the convalescent plasma is collected that the donors need to go to, with a typical such destination location denoted by $j$. As mentioned in the Introduction, there are different types of products that can be produced from the donated convalescent plasma, including that for transfusion into Covid-19 patients. We denote a convalescent plasma product by $l$ and there are $r$ such products. Also, there are $o$ different classes of convalescent plasma donors, with a typical class denoted by $k$. The network structure for the model is shown in Fig. 1.

All vectors are column vectors. The notation for the model is given in Table 1 . Note that we assume that there is a utility function associated with each donor class located at a location and associated with a convalescent plasma product and donation location. In addition, since convenience, safety, risk, time, familiarity, and comfort with a collection site, and possible monetary expenditure are important, we associate a generalized cost for each class at each location in donating for a specific convalescent plasma product at a particular donor site.

The convalescent plasma donations must be nonnegative, that is,

$$
Q_{i j}^{k l} \geq 0, \quad \forall i, j, k, l .
$$

It is to be noted that the amount of plasma that can be collected from each donor depends on their height, weight, and gender [88]. The flows here denote the number of units of convalescent plasma donated by each class of donors from each location to an organization for a particular purpose.

The convalescent plasma donors seek to identify the product towards which their convalescent plasma will be used as well as the location for their donations. There

Fig. 1 Donor Model

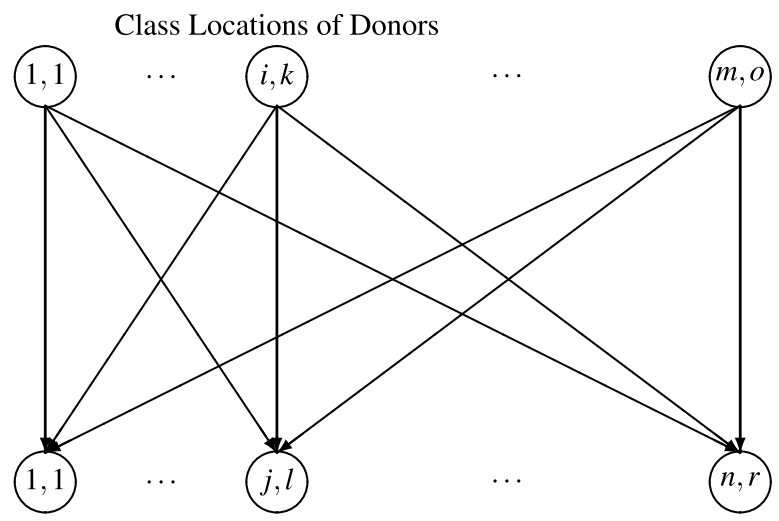

Plasma Collection Site Locations for Products 
Table 1 Notation for the Multiclass, Multiproduct Convalescent Plasma Donor Model

\begin{tabular}{|c|c|}
\hline Notation & Definition \\
\hline$Q_{i j}^{k l}$ & $\begin{array}{l}\text { the donation flow of convalescent plasma for purpose } l \text { of class } k \text { donor from location } i \text { to } \\
\text { collection site } j \text {. The }\left\{Q_{i j}^{k l}\right\} \text { elements for all } i, j, k \text {, and } l \text { are grouped into the vector } Q \in R_{+}^{m n o r} \text {. }\end{array}$ \\
\hline $\bar{Q}_{i}^{k}$ & $\begin{array}{l}\text { the nonnegative amount of convalescent plasma from potential donors of class } k \text { at location } i \text {; } \\
\qquad k=1, \ldots, o ; i=1, \ldots, m \text {. }\end{array}$ \\
\hline$U_{i j}^{k l}(Q)$ & $\begin{array}{l}\text { the utility perceived by class } k \text { at location } i \text { to donate convalescent plasma at location } j \text { for } \\
\text { product/purpose } l ; i=1, \ldots, m ; j=1, \ldots, n ; k=1, \ldots, o ; l=1, \ldots, r \text {. We group all the utili- } \\
\text { ties into the vector } U(Q) \in R^{m n o r} \text {. }\end{array}$ \\
\hline$c_{i j}^{k l}(Q)$ & $\begin{array}{l}\text { the generalized cost of class } k \text { at location } i \text { to go to location } j \text { to donate product } l \text { of their } \\
\text { convalescent plasma, which includes financial cost, time, and risk of class } k \text { for } i=1, \ldots, m \text {; } \\
j=1, \ldots, n ; k=1, \ldots, o ; l=1, \ldots, r \text {. We group all the generalized costs into the vector } \\
c(Q) \in R^{\text {mnor }} \text {. }\end{array}$ \\
\hline$\lambda_{i}^{k}$ & $\begin{array}{l}\text { the nonnegative Lagrange multiplier, in effect, associated with the available amount of } \\
\text { convalescent plasma potentially to donate by class } k \text { located at location } i ; i=1, \ldots, m \text {; } \\
k=1, \ldots, o \text {. We group all the Lagrange multipliers into the vector } \lambda \in R_{+}^{o m} \text {. }\end{array}$ \\
\hline
\end{tabular}

is a fixed amount of convalescent plasma $\bar{Q}_{i}^{k}$ in the recovered population at a location $i$ and class $k$. The equilibrium conditions which reflect the choices made by the convalescent plasma donors guarantee that if the cost exceed the utility, then the associated flow of convalescent plasma donations will be zero. Also, since the convalescent plasma donor population may not be sufficiently high to clear the market, there is a Lagrange multiplier associated with a class and origin location that will be positive, in equilibrium, if the market clears, and zero, otherwise.

We assume that the utility functions and the generalized cost functions are continuous with the former being monotonically decreasing in $\mathrm{Q}$ and the latter-monotonically increasing. It makes sense to have these cost functions be monotone increasing since the greater the volume of those moving from an origin node to a donor destination node, the greater the chance of contagion of various diseases and other associated risks; the longer it can take to get to the donor site and to get processed (which would include the waiting time), and it also "costs" more due to the opportunity cost, the cost of gas or using public transportation, etc. On the other hand, having the utility functions be monotone decreasing is not unreasonable due to analogues of our framework with models of human migration networks, where monotone decreasing utility functions have been historically utilized in the literature.

Note that we allow the utility functions and the generalized cost functions to depend upon, in general, vectors of variables. Of course, a special case would be that of separable functions. We emphasize that one can expect that the utility of a donor in a class at a donation node would clearly depend on the volume of donors not only of the same class but of other classes. Also, if the donor sites are in proximity, one can expect some interaction. In fact, the role played by word-of-mouth in charitable donations has been studied in the existing literature. Sundermann [89] reported the positive impact of word-of-mouth on commitment, satisfaction, and loyalty of blood donors. Martin et al. [90] examined survey data on blood donors of the Austrian Red Cross and found evidence to support that a strong relationship exists between wordof-mouth and awareness of blood services and willingness to donate. Williams and 
Buttle [91] also point out the important role played by word-of-mouth in the case of donor behavior and donations to nonprofit organizations. Hence, it is reasonable to assume that the utility perceived by donors is influenced and affected by other classes of donors donating at the same location and/or those who choose to donate plasma to other locations. Clearly, the generalized costs are also influenced by the volume of flow of donors not only of the same class but also of different classes on a link, and, very likely, at the very least of neighboring links.

We define the feasible set $K \equiv\left\{(Q, \lambda) \mid\right.$ such that $Q \in R_{+}^{\text {mnor }}$ and $\left.\lambda \in R_{+}^{\text {om }}\right\}$. Note that the feasible set $K$ is closed and convex.

The full statement of the governing equilibrium conditions is below.

Definition 1 The Multiclass, Multiproduct Convalescent Plasma Donor Equilibrium Conditions

A vector of multiclass, multiproduct convalescent plasma donations (flows) and a vector of Lagrange multipliers $\left(Q^{*}, \lambda^{*}\right) \in K$ are in equilibrium if they satisfy the equilibrium conditions: for each class $k ; k=1, \ldots, o$ and each product $l ; l=1, \ldots, r$ :

$$
c_{i j}^{k l}\left(Q^{*}\right)\left\{\begin{array}{lll}
=U_{i j}^{k l}\left(Q^{*}\right)-\lambda_{i}^{k *}, & \text { if } & Q_{i j}^{k l *}>0, \\
\geq U_{i j}^{k l}\left(Q^{*}\right)-\lambda_{i}^{k *}, & \text { if } & Q_{i j}^{k l *}=0,
\end{array}\right.
$$

and

$$
\bar{Q}_{i}^{k}\left\{\begin{array}{lll}
=\sum_{j=1}^{n} \sum_{l=1}^{r} Q_{i j}^{k l *}, & \text { if } & \lambda_{i}^{k *}>0, \\
\geq \sum_{j=1}^{n} \sum_{l=1}^{r} Q_{i j}^{k l *}, & \text { if } & \lambda_{i}^{k *}=0 .
\end{array}\right.
$$

We now provide a deeper interpretation of equilibrium conditions (2). Specifically, we note that they can be rewritten as follows: for each class $k ; k=1, \ldots, o$ and each product $l ; l=1, \ldots, r$ :

$$
\lambda_{i}^{k *} \begin{cases}=U_{i j}^{k l}\left(Q^{*}\right)-c_{i j}^{k l}\left(Q^{*}\right), & \text { if } \quad Q_{i j}^{k l *}>0, \\ \geq U_{i j}^{k l}\left(Q^{*}\right)-c_{i j}^{k l}\left(Q^{*}\right), & \text { if } \quad Q_{i j}^{k l *}=0 .\end{cases}
$$

From (4) we can see that, for a given class at an origin location, the utility minus the generalized cost is equalized for all plasma product / destination choices that are selected, that is, for those for which there is a positive volume of convalescent plasma donated. And that difference between the utility and the generalized cost exceeds the analogous values for the not selected options, that is, those with zero convalescent plasma donations (flows).

We now provide the variational inequality (VI) formulation of the equilibrium conditions (2) and (3) in the following theorem.

\section{Theorem 1 Variational Inequality Formulation}

The vectors of convalescent plasma donations (flows) and Lagrange multipliers satisfy the equilibrium conditions (2) and (3) if and only if they satisfy the variational inequality problem: determine $\left(Q^{*}, \lambda^{*}\right) \in K$ such that 


$$
\begin{gathered}
\sum_{i=1}^{m} \sum_{j=1}^{n} \sum_{k=1}^{o} \sum_{l=1}^{r}\left[c_{i j}^{k l}\left(Q^{*}\right)-U_{i j}^{k l}\left(Q^{*}\right)+\lambda_{i}^{k *}\right] \times\left[Q_{i j}^{k l}-Q_{i j}^{k l *}\right] \\
+\sum_{i=1}^{m} \sum_{k=1}^{o}\left[\bar{Q}_{i}^{k}-\sum_{j=1}^{n} \sum_{l=1}^{r} Q_{i j}^{k l *}\right] \times\left[\lambda_{i}^{k}-\lambda_{i}^{k *}\right] \geq 0, \quad \forall(Q, \lambda) \in K .
\end{gathered}
$$

Proof We first establish necessity, that is, we show that if $\left(Q^{*}, \lambda^{*}\right) \in K$ satisfies equilibrium conditions (2) and (3), then it also satisfies VI (5).

If $\left(Q^{*}, \lambda^{*}\right) \in K$ satisfies (2), then, for a fixed $i, j, k, l$, we know that

$$
\left[c_{i j}^{k l}\left(Q^{*}\right)-U_{i j}^{k l}\left(Q^{*}\right)+\lambda_{i}^{k *}\right] \times\left[Q_{i j}^{k l}-Q_{i j}^{k l *}\right] \geq 0, \quad \forall Q_{i j}^{k l} \geq 0 .
$$

Indeed, if $Q_{i j}^{k l *}>0$, then the above holds true and the same for $Q_{i j}^{k l *}=0$.

Summing (6) over all $i, j, k, l$, we have that

$$
\sum_{i=1}^{m} \sum_{j=1}^{n} \sum_{k=1}^{o} \sum_{l=1}^{r}\left[c_{i j}^{k l}\left(Q^{*}\right)-U_{i j}^{k l}\left(Q^{*}\right)+\lambda_{i}^{k *}\right] \times\left[Q_{i j}^{k l}-Q_{i k}^{k l *}\right] \geq 0, \quad \forall Q \in R_{+}^{\text {mnor }} .
$$

Similarly, we know from equilibrium conditions (3), that, for a fixed $i$ and $k$ :

$$
\left[\bar{Q}_{i}^{k}-\sum_{j=1}^{n} \sum_{l=1}^{r} Q_{i j}^{k l *}\right] \times\left[\lambda_{i}^{k}-\lambda_{i}^{k *}\right] \geq 0, \quad \forall \lambda_{i}^{k} \geq 0 .
$$

Since (8) holds for any $i, k$, summation of (8) over all $i, k$ yields:

$$
\sum_{i=1}^{m} \sum_{k=1}^{o}\left[\bar{Q}_{i}^{k}-\sum_{j=1}^{n} \sum_{l=1}^{r} Q_{i j}^{k l *}\right] \times\left[\lambda_{i}^{k}-\lambda_{i}^{k *}\right] \geq 0, \quad \forall \lambda \in R_{+}^{m o} .
$$

Finally, summation of inequalities (7) and (9) yields VI (5).

We now establish sufficiency, that is, a solution $\left(Q^{*}, \lambda^{*}\right) \in K$ to VI (5), also satisfies equilibrium conditions (2) and (3).

In (5), we make the following substitutions: we set $\lambda_{q}^{s}=\lambda_{q}^{s *}$ for all $q \neq i$ and $s \neq k$ and $Q_{i j}^{k l}=Q_{i j}^{k l *}$, for all $i, j, k, l$. This results in:

$$
\left[\bar{Q}_{i}^{k}-\sum_{j=1}^{n} \sum_{l=1}^{r} Q_{i j}^{k l *}\right] \times\left[\lambda_{i}^{k}-\lambda_{i}^{k *}\right] \geq 0, \quad \forall \lambda_{i}^{k} \geq 0,
$$

which implies that equilibrium condition (3) holds.

Now, we set $\lambda_{i}^{k}=\lambda_{i}^{k *}$ for all $i, k, \quad$ in VI (5) and we set $Q_{q t}^{s h}=Q_{q t}^{s h *}$ for all $q, t, s, h \neq i, j, k, l$ and substitute into VI (5), which yields:

$$
\left[c_{i j}^{k l}\left(Q^{*}\right)-U_{i j}^{k l}\left(Q^{*}\right)+\lambda_{i}^{k *}\right] \times\left[Q_{i j}^{k l}-Q_{i j}^{k l *}\right] \geq 0, \quad \forall Q_{i j}^{k l} \geq 0
$$

and inequality (11) clearly implies that equilibrium condition (2) holds. The proof is complete. 


\subsection{An Extension to Include Capacities}

It is important to recognize that some collection sites may be capacitated. Hence, we introduce some new notation. We let cap $_{j}$ denote the maximum amount of plasma donations that can be handled at collection site $j$ and we let $\mathcal{S}$ be the set of such sites $j$. We assume that there are $n_{S}$ elements in the set $\mathcal{S}$. Also, we introduce the Lagrange multiplier $\gamma_{j}$ for each collection site $j \in \mathcal{S}$ and we group the Lagrange multipliers $\gamma_{j}$ into the vector $\gamma \in R_{+}^{n_{S}}$. We define the feasible set $K^{2} \equiv\left\{(Q, \lambda, \gamma) \mid\right.$ such that $\left.Q \in R_{+}^{\text {mnor }}, \lambda \in R_{+}^{\text {om }}, \gamma \in R_{+}^{n_{S}}\right\}$.

In the capacitated extension of the above model, the equilibrium conditions are now as below.

Definition 2 The Capacitated Multiclass, Multiproduct Convalescent Plasma Donor Equilibrium Conditions

A vector of multiclass, multiproduct convalescent plasma donations (flows) and vectors of Lagrange multipliers $\left(Q^{*}, \lambda^{*}, \gamma^{*}\right) \in K^{2}$ are in equilibrium if they satisfy the equilibrium conditions: for each class $k ; k=1, \ldots, o$, each product $l ; l=1, \ldots, r$, for donors at $i ; i=1, \ldots, m$, and for $j \in \mathcal{S}$ :

$$
c_{i j}^{k l}\left(Q^{*}\right)\left\{\begin{array}{lll}
=U_{i j}^{k l}\left(Q^{*}\right)-\lambda_{i}^{k *}-\gamma_{j}^{*}, & \text { if } & Q_{i j}^{k l *}>0, \\
\geq U_{i j}^{k l}\left(Q^{*}\right)-\lambda_{i}^{k *}-\gamma_{j}^{*}, & \text { if } \quad & Q_{i j}^{k l *}=,
\end{array}\right.
$$

whereas for each class $k ; k=1, \ldots, o$, each product $l ; l=1, \ldots, r$, for donors at $i$; $i=1, \ldots, m$, and for $j \notin \mathcal{S}$ :

$$
c_{i j}^{k l}\left(Q^{*}\right)\left\{\begin{array}{lll}
=U_{i j}^{k l}\left(Q^{*}\right)-\lambda_{i}^{k *}, & \text { if } & Q_{i j}^{k l *}>0, \\
\geq U_{i j}^{k l}\left(Q^{*}\right)-\lambda_{i}^{k *}, & \text { if } & Q_{i j}^{k l *}=0,
\end{array}\right.
$$

and for $j \in \mathcal{S}$ :

$$
\operatorname{cap}_{j}\left\{\begin{array}{lll}
=\sum_{i=1}^{m} \sum_{k=1}^{o} \sum_{l=1}^{r} Q_{i j}^{k l *}, \text { if } & \gamma_{j}^{*}> \\
\geq \sum_{i=1}^{m} \sum_{k=1}^{o} \sum_{l=1}^{r} Q_{i j}^{k l *}, \text { if } & \gamma_{j}^{*}=0,
\end{array}\right.
$$

with (3), as before, holding for all $j$, that is,

$$
\bar{Q}_{i}^{k} \begin{cases}=\sum_{j=1}^{n} \sum_{l=1}^{r} Q_{i j}^{k l *}, \text { if } & \lambda_{i}^{k *}>0 \\ \geq \sum_{j=1}^{n} \sum_{l=1}^{r} Q_{i j}^{k l *}, \text { if } & \lambda_{i}^{k *}=0\end{cases}
$$

The following variational inequality formulation of the above equilibrium conditions is immediate.

Theorem 2 Variational Inequality Formulation of the Capacitated Model

A vector of multiclass, multiproduct convalescent plasma donations (flows) and a vector of Lagrange multipliers $\left(Q^{*}, \lambda^{*}, \gamma^{*}\right) \in K^{2}$ satisfy the equilibrium conditions 
(12), (13), (14), and (15) if and only if they satisfy the variational inequality problem: determine $\left(Q^{*}, \lambda^{*}, \gamma^{*}\right) \in K^{2}$ such that

$$
\begin{aligned}
& \sum_{i=1}^{m} \sum_{j \in S} \sum_{k=1}^{o} \sum_{l=1}^{r}\left[c_{i j}^{k l}\left(Q^{*}\right)-U_{i j}^{k l}\left(Q^{*}\right)+\lambda_{i}^{k *}+\gamma_{j}^{*}\right] \times\left[Q_{i j}^{k l}-Q_{i j}^{k l *}\right] \\
& +\sum_{i=1}^{m} \sum_{j \notin S} \sum_{k=1}^{o} \sum_{l=1}^{r}\left[c_{i j}^{k l}\left(Q^{*}\right)-U_{i j}^{k l}\left(Q^{*}\right)+\lambda_{i}^{k *}\right] \times\left[Q_{i j}^{k l}-Q_{i j}^{k l *}\right] \\
& +\sum_{j \in S}\left[\operatorname{cap}_{j}-\sum_{i=1}^{m} \sum_{k=1}^{o} \sum_{l=1}^{r} Q_{i j}^{k l *}\right] \times\left[\gamma_{j}-\gamma_{j}^{*}\right] \\
& +\sum_{i=1}^{m} \sum_{k=1}^{o}\left[\bar{Q}_{i}^{k}-\sum_{j=1}^{n} \sum_{l=1}^{r} Q_{i j}^{k l *}\right] \times\left[\lambda_{i}^{k}-\lambda_{i}^{k *}\right] \geq 0, \quad \forall\left(Q^{*}, \lambda^{*}, \gamma^{*}\right) \in K^{2} .
\end{aligned}
$$

We now put variational inequality (5) into standard form (cf. Nagurney [66]): determine $X^{*} \in \mathcal{K}$ such that

$$
\left\langle F\left(X^{*}\right), X-X^{*}\right\rangle \geq 0, \quad \forall X \in \mathcal{K},
$$

where $F$ is a given continuous function from $\mathcal{K}$ to $R^{N}, \mathcal{K}$ is a given closed convex set, and $\langle\cdot, \cdot\rangle$ denotes the inner product in $N$-dimensional Euclidean space.

We set $\mathcal{K} \equiv K, X \equiv(Q, \lambda)$, and $N=$ mnor $+m o$. Also, we define the vector $F \equiv\left(F_{1}, F_{2}\right)$, where the components of $F_{1}$ consist of the elements: $c_{i j}^{k l}(Q)-U_{i j}^{k l}(Q)+\lambda_{i}^{k}$, $\forall i, j, k, l$, and the components of $F_{2}$ consist of the elements: $\bar{Q}_{i}^{k}-\sum_{j=1}^{n} \sum_{l=1}^{r} Q_{i j}^{k l}, \forall i, k$. Then, clearly, VI (5) coincides with VI (17), with the above definitions.

VI (16) can, analogously, also be put into standard form (17), but with the expansion of the definitions of the vectors $X$ and $F(X)$ and also of $R^{N}$ since in the constrained case we have additional Lagrange multipliers in the vector $\gamma$.

\section{Qualitative Properties and the Algorithm}

We now discuss some properties of the model, specifically, those that guarantee that the conditions for convergence of the modified projection method (cf. Korpelevich [92] and Nagurney [66]) that we use to compute solutions to numerical examples in this next section are met. Specifically, the algorithm is guaranteed to converge to a solution of variational inequality (17) if the function $F(X)$ that enters the VI is monotone and Lipschitz continuous, and that a solution exists. It was recently applied to compute solutions to a stochastic game theory model for disaster relief by [93]. 
Recall that the function $F(X)$ is said to be monotone, if

$$
\left\langle F\left(X^{1}\right)-F\left(X^{2}\right), X^{1}-X^{2}\right\rangle \geq 0, \quad \forall X^{1}, X^{2} \in \mathcal{K} .
$$

The function $F(X)$ is Lipschitz continuous, if there exists a constant $L>0$, known as the Lipschitz constant, such that

$$
\left\|F\left(X^{1}\right)-F\left(X^{2}\right)\right\| \leq L\left\|X^{1}-X^{2}\right\|, \quad \forall X^{1}, X^{2} \in \mathcal{K} .
$$

We now write $\left\langle F\left(X^{1}\right)-F\left(X^{2}\right), X^{1}-X^{2}\right\rangle$ for $F(X)$ as defined for VI (5) and obtain, after algebraic simplification:

$$
\sum_{i=1}^{m} \sum_{j=1}^{n} \sum_{k=1}^{o} \sum_{l=1}^{r}\left(\left(c_{i j}^{k l}\left(Q^{1}\right)-U_{i j}^{k l}\left(Q^{2}\right)\right)-\left(c_{i j}^{k l}\left(Q^{2}\right)-U_{i j}^{k l}\left(Q^{2}\right)\right) \times\left(Q_{i j}^{k l 1}-Q_{i j}^{k l 2}\right) .\right.
$$

And, under our previously imposed assumptions that $c(Q)$ is monotone increasing and that $U(Q)$ is monotone decreasing it follows that the term in (19) is greater than or equal to zero for all $\left(Q^{1}, Q^{2}\right) \in K$, and, hence, $F(X)$ is monotone. Under the same assumptions, the $F(X)$ for VI (15) is also monotone.

As for Lipschitz continuity, if both the generalized cost and the utility functions have bounded second order partial derivatives, then $F(X)$ will be Lipschitz continuous (see also Nagurney and Zhang [94] and Nagurney [66]).

The iterative steps of the modified projection method, with $\tau$ denoting an iteration counter, are as follows:

\section{The Modified Projection Method}

Step 0: Initialization

Initialize with $X^{0} \in \mathcal{K}$. Set the iteration counter $\tau:=1$ and let $\beta$ be a scalar such that $0<\beta \leq \frac{1}{L}$, where $L$ is the Lipschitz constant.

\section{Step 1: Computation}

Compute $\bar{X}^{\tau}$ by solving the variational inequality subproblem:

$$
\left\langle\bar{X}^{\tau}+\beta F\left(X^{\tau-1}\right)-X^{\tau-1}, X-\bar{X}^{\tau}\right\rangle \geq 0, \quad \forall X \in \mathcal{K} .
$$

\section{Step 2: Adaptation}

Compute $X^{\tau}$ by solving the variational inequality subproblem:

$$
\left\langle X^{\tau}+\beta F\left(\bar{X}^{\tau}\right)-X^{\tau-1}, X-X^{\tau}\right\rangle \geq 0, \quad \forall X \in \mathcal{K} .
$$

\section{Step 3: Convergence Verification}

If $\left|X^{\tau}-X^{\tau-1}\right| \leq \epsilon$, with $\epsilon>0$, a pre-specified tolerance, then stop; otherwise, set $\tau:=\tau+1$ and go to Step 1 .

The modified projection method for both the model governed by VI (5) and that capacitated extension one governed by VI (16) yields closed form expressions for the convalescent plasma product donations and for the Lagrange multipliers in both Steps (21) and (22). hence, these are nice features for implementation. 


\section{Numerical Examples}

In this Section, we apply the modified projection method to compute solutions to numerical examples. The algorithm was implemented in FORTRAN and the computer system used was a Linux system at the University of Massachusetts Amherst. We initialized the algorithm by setting all the donation flows and the Lagrange multipliers to 0.00 . The convergence condition for all the examples was that the absolute value of two successive variable iterates was less than or equal to $10^{-7}$.

The model developed in this paper can be applied to any region or country that is recruiting convalescent plasma donors. The examples here, although stylized, are inspired by the Covid-19 outbreak in New York City, the areas that have been affected, the number of recovered patients, and also the availability of collection sites for convalescent plasma products as of May 2020. We assume the time frame for the model to be a month. Hence, the flows obtained here denote the number of units of convalescent plasma donated in a month. The American Red Cross which began collection with FDA's approval in early April reportedly had collected around 200 units of convalescent plasma towards the end of the month [95]. The values we obtain from the numerical examples are close to the above figure.

The series of numerical examples are constructed in increasing order of complexity.

\section{Example 1: Single Class, Two Origin Locations, and a Single Nonprofit Collection Location}

The first example consists of a single class of donor at two locations: Williamsburg, Brooklyn, corresponding to origin location 1 and Corona, Queens, corresponding to origin location 2 . These location areas are among the most severely affected neighborhoods in New York [25, 96]. There is a single collection site for convalescent plasma as depicted in Fig. 2 which we associate with a nonprofit organization such as Mount Sinai Hospital which spearheaded the convalescent plasma treatment in New York City [76].

For completeness, and easy reference, we also map the donation flow variable with the numbered link in Fig. 2. Specifically, $Q_{11}^{11}$ is the flow on link 1 and $Q_{21}^{11}$ is the flow on link 2 . Recall that we use $Q_{i j}^{k l}$ notation for the convalescent plasma donations of those located at origin location $i$ of class $k$ donating at collection site $j$ for use in product $l$.

The available supplies (bounds) at the origin locations of the convalescent plasma that can be donated are:

$$
\bar{Q}_{1}^{1}=100.00, \quad \bar{Q}_{2}^{1}=150.00 .
$$

The generalized cost functions are: 
Fig. 2 Network Topology for Example 1

\section{Class Locations of Donors}

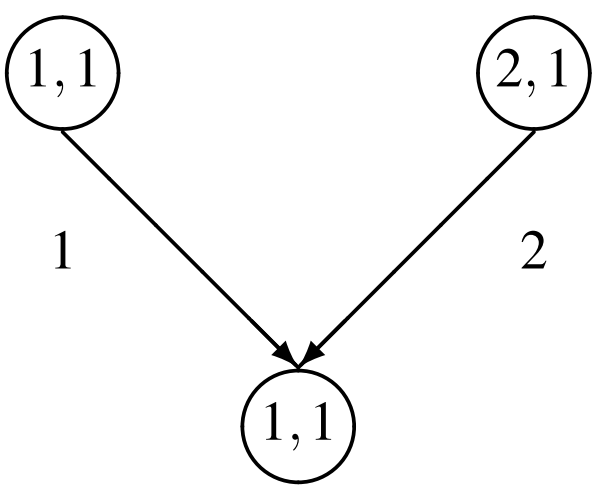

\section{Sites for Plasma Collection}

$$
c_{11}^{11}(Q)=2 Q_{11}^{11}+2, \quad c_{21}^{11}(Q)=3 Q_{21}^{11}+3 .
$$

The utility functions are:

$$
U_{11}^{11}(Q)=-1 Q_{11}^{11}+1000, \quad U_{21}^{11}(Q)=-1 Q_{21}^{11}+1500 .
$$

The modified projection method converged to the following equilibrium solution:

$$
Q_{11}^{11 *}=100.00, \quad Q_{21}^{11 *}=150.00,
$$

with

$$
\lambda_{1}^{1 *}=698.00, \quad \lambda_{2}^{1 *}=897.00 .
$$

The utilities at the equilibrium solution are:

$$
U_{11}^{11}\left(Q^{*}\right)=900.00, \quad U_{21}^{11}\left(Q^{*}\right)=1350.00 .
$$

In this example, we can see that the donors at both origin locations choose to donate all their available convalescent plasma. The Lagrange multipliers are, hence, positive. We are seeing this in practice, that those who have recovered from Covid-19 are seeking to give back and help others through donations of convalescent plasma.

\section{Example 2: Single Class, Two Origin Locations, and Two Nonprofit Collection Locations}

Example 2 is constructed from Example 1 and has the same data but with the addition of new data to handle a new collection site for convalescent plasma for another nonprofit. This new collection site, at destination location 2, is perceived as the New 
Fig. 3 Network Topology for Example 2
Class Locations of Donors

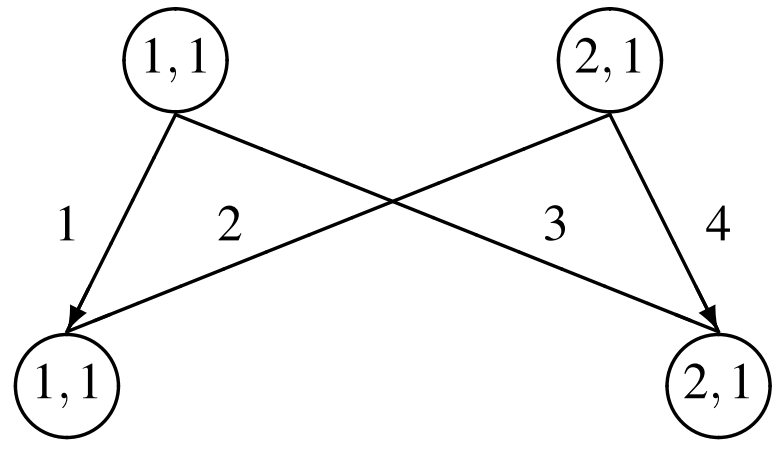

Sites for Plasma Collection

York Blood Center site in Long Island City which is closer to the donor origin locations. The network topology for this example is depicted in Fig. 3.

Note that new links 3 and 4 have been added joining the origin locations with the new collection site in Fig. 3. The plasma donation variable associated with link 3 is: $Q_{12}^{11}$, whereas that for link 4 is: $Q_{22}^{11}$.

The generalized cost functions associated with the added collection site (and the new links) are:

$$
c_{12}^{11}(Q)=2 Q_{12}^{11}+1, \quad c_{22}^{11}(Q)=4 Q_{22}^{11}+2 .
$$

The utility functions, in turn, are

$$
U_{12}^{11}(Q)=-2 Q_{12}^{11}+2000, \quad U_{22}^{11}(Q)=-1 Q_{22}^{11}+2000 .
$$

The utility functions have higher fixed terms at the new collection site than at the first one since the class of donors feels that the specialization in blood services is a positive feature of the site. Since there could be traffic, even in the pandemic, this is captured by a higher coefficient of " 4 " preceding the donation flow $Q_{22}^{11}$.

The modified projection method converged to the following equilibrium solution:

$$
Q_{11}^{11 *}=0.00, \quad Q_{21}^{11 *}=27.67, \quad Q_{12}^{11 *}=100.00, \quad Q_{22}^{11 *}=122.33,
$$

and

$$
\lambda_{1}^{1 *}=1599.00, \quad \lambda_{2}^{1 *}=1386.33 .
$$

The utilities at the equilibrium solution are:

$$
U_{11}^{11}\left(Q^{*}\right)=1000.00, U_{21}^{11}\left(Q^{*}\right)=1472.33, U_{12}^{11}\left(Q^{*}\right)=1800.00, U_{22}^{11}\left(Q^{*}\right)=1877.67 .
$$


Observe that with the new collection site, the donors at the first origin location now donate all their convalescent plasma to the new collection site. Also, the donors at the second origin site donate the majority of their plasma to the new site. Again, all the available plasma is donated and the Lagrange multipliers are positive.

\section{Example 3 - Single Class, Two Origin Locations, and Three Collection Locations, with One Being a For Profit One Collecting for Another Product}

Example 3 has the same data as Example 2 but now we consider the following scenario. A for-profit organization such as Grifols or Takeda has heard of the number of convalescent plasma donations at the second collection point and has decided to locate very near there. The convalescent plasma will not be for use soon thereafter in patients but, rather, for a new product. The new collection site 3 and the associated links (links 5 and 6) destined to it are depicted in the network topology representing this example in Fig. 4. The plasma donation variable associated with link 5 is: $Q_{13}^{12}$ and that with link 6: $Q_{23}^{12}$.

The data for Example 3 was the same as that for Example 2 with the following additions for the new collection site which collects for a different product geared to making a profit.

The added generalized cost functions associated with the new collection site associated with a for-profit organization are:

$$
c_{13}^{12}(Q)=2 Q_{13}^{12}+1, \quad c_{23}^{12}(Q)=4 Q_{23}^{12}+2 .
$$

The added utility functions, in turn, are:

$$
U_{13}^{12}(Q)=-1 Q_{13}^{12}+2500, \quad U_{23}^{12}(Q)=-1 Q_{23}^{12}+2500 .
$$

Note that the utility function fixed terms associated with donating to the new collection site are higher than those fixed terms associated with the other nonprofit collection sites since donors now get financial compensation for their convalescent plasma donations at the third destination site. Also, note that the analogous generalized cost

Fig. 4 Network Topology for Example 3
Class Locations of Donors

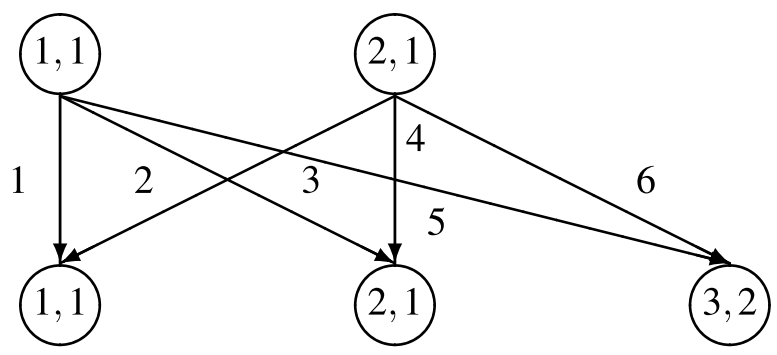

Sites for Plasma Collection 
functions are the same since the location of the collection site is essentially at the same place as the second collection site.

The modified projection method converged to the following equilibrium solution:

$$
\begin{gathered}
Q_{11}^{11 *}=0.00, \quad Q_{21}^{11 *}=0.00, \quad Q_{12}^{11 *}=0.00, \quad Q_{22}^{11 *}=25.00 \\
Q_{13}^{12 *}=100.00, \quad Q_{23}^{12 *}=125.00
\end{gathered}
$$

and

$$
\lambda_{1}^{1 *}=2199.00, \quad \lambda_{2}^{1 *}=1873.00
$$

The utilities at the equilibrium solution are:

$$
\begin{gathered}
U_{11}^{11}\left(Q^{*}\right)=1000.00, U_{21}^{11}\left(Q^{*}\right)=1500.00, U_{12}^{11}\left(Q^{*}\right)=2000.00, U_{22}^{11}\left(Q^{*}\right)=1975.00, \\
U_{13}^{12}\left(Q^{*}\right)=2400.00, \quad U_{23}^{12}\left(Q^{*}\right)=2375.00 .
\end{gathered}
$$

The donors at origin location 1 now donate all their convalescent plasma to the for profit collection site. The donors at the second origin location donate the majority of their convalescent plasma also to the for profit collection site, and a much smaller amount to the second collection site. Clearly, the nonprofits lose in terms of convalescent plasma donations due to the competition from the profit organization.

\section{Example 4 - Variant of Example 3}

In Example 4, we consider the following scenario. The donors at the second origin location are now a bit embarrassed by those in their community in that they are donating so much convalescent plasma to the for-profit organization.

Hence, their utility function associated with donating to the for profit has now been modified to:

$$
U_{23}^{12}(Q)=-1 Q_{23}^{12}-.7 Q_{22}^{11}+2500 .
$$

The modified utility function reflects that the utility function is also decreasing in the number of convalescent plasma donations from that origin location to the second donation site.

The modified projection method now yielded to the following equilibrium solution:

$$
\begin{gathered}
Q_{11}^{11 *}=0.00, \quad Q_{21}^{11 *}=0.00, \quad Q_{12}^{11 *}=0.00, \quad Q_{22}^{11 *}=26.88 \\
Q_{13}^{12 *}=100.00, \quad Q_{23}^{12 *}=123.12
\end{gathered}
$$


and

$$
\lambda_{1}^{1 *}=2199.00, \quad \lambda_{2}^{1 *}=1863.59 .
$$

Once can now that there is a positive amount of convalescent plasma that is being "switched" from being donated to the for-profit organization to the nonprofit one at the second collection site. All the plasma at both origin locations is fully donated with the Lagrange multipliers, again, being both positive.

The utilities at the equilibrium solution are now:

$$
\begin{gathered}
U_{11}^{11}\left(Q^{*}\right)=1000.00, U_{21}^{11}\left(Q^{*}\right)=1500.00, U_{12}^{11}\left(Q^{*}\right)=2000.00, U_{22}^{11}\left(Q^{*}\right)=1973.12, \\
U_{13}^{12}\left(Q^{*}\right)=2400.00, \quad U_{23}^{12}\left(Q^{*}\right)=2358.06 .
\end{gathered}
$$

\section{Example 5 and Variant - Addition of a New Class}

Example 5 has the same data as Example 4 but now we introduce a new class 2 that, because of specific criteria that must be satisfied, is not able to donate their convalescent plasma for direct infusion into Covid-19 patients but, rather, can still donate for another product and that product is managed by the for-profit organization at its collection site 3. The network topology is as in Fig. 5. The new link is link 7 and it is associated with the plasma donation variable: $Q_{23}^{22}$.

The additional data associated with link 7 are as follows.

The generalized link cost is:

$$
c_{23}^{22}(Q)=6 Q_{23}^{22}+5
$$

and the utility function is:

$$
U_{23}^{22}(Q)=-1 Q_{23}^{22}+.4 Q_{23}^{12}+2000 .
$$

Also, we have:

Fig. 5 Network Topology for Example 5
Class Locations of Donors

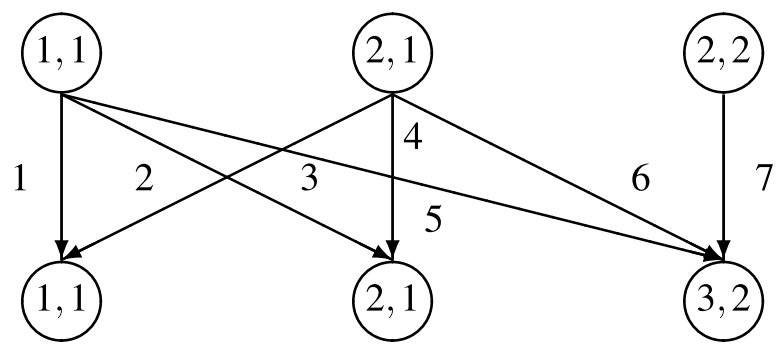

Sites for Plasma Collection 


$$
\bar{Q}_{2}^{2}=140.00 .
$$

The above generalized link cost captures culture barriers in that this class may be reluctant to travel, whereas the utility function captures that there is a positive effect, due to familiarity, associated with the other class from the same origin location donating to the for profit collection site.

The modified projection method yielded the equilibrium solution:

$$
\begin{gathered}
Q_{11}^{11 *}=0.00, \quad Q_{21}^{11 *}=0.00, \quad Q_{12}^{11 *}=0.00, \quad Q_{22}^{11 *}=26.88, \\
Q_{13}^{12 *}=100.00, \quad Q_{23}^{12 *}=123.12, \quad Q_{23}^{22 *}=140.00,
\end{gathered}
$$

and

$$
\lambda_{1}^{1 *}=2199.00, \quad \lambda_{2}^{1 *}=1864.00, \quad \lambda_{2}^{2 *}=1064.24 .
$$

The new class of donor donates its entire supply of convalescent plasma and the equilibrium Lagrange multiplier is positive.

The utilities at the equilibrium solution are now:

$$
\begin{gathered}
U_{11}^{11}\left(Q^{*}\right)=1000.00, U_{21}^{11}\left(Q^{*}\right)=1500.00, U_{12}^{11}\left(Q^{*}\right)=2000.00, U_{22}^{11}\left(Q^{*}\right)=1973.12, \\
U_{12}^{13}\left(Q^{*}\right)=2400.00, \quad U_{23}^{12}\left(Q^{*}\right)=2358.06, \quad U_{23}^{22}\left(Q^{*}\right)=1909.25 .
\end{gathered}
$$

We then modified the new class donors' utility function to signify a decrease in utility associated with donating as follows:

$$
U_{23}^{22}(Q)=-1 Q_{23}^{22}+.4 Q_{23}^{12}+500 .
$$

The modified projection method converged to an equilibrium solution, which differed from the one immediately above in that:

$$
Q_{23}^{22 *}=77.75, \quad \lambda_{2}^{2 *}=0.00
$$

with $U_{23}^{22}\left(Q^{*}\right)=471.50$.

Observe that now class 2 does not donate all of the convalescent plasma that it can and, hence, the associated Lagrange multiplier is equal to 0.00 .

\section{Example 6 - Bound On Location 3 Collection Site}

Example 6 was inspired by the following scenario. The workers at collection site 3 have been falling ill with Covid-19 and there are, hence, fewer labor resources available for handling the convalescent plasma donations at this for-profit organization collection site.

The solutions to all the preceding examples satisfied the unconstrained version VI (5), whereas the governing equilibrium conditions for this constrained example 
satisfy VI (15), where recall that we have an additional Lagrange multiplier $\gamma$ associated with the bound on the flows to the destination location under the capacity constraint.

There is, hence, a capacity at this site of: $\mathrm{cap}_{3}=300.00$, which is imposed on donations/flows to node $(3,2)$ in Fig. 5. Hence, links 5, 6, and 7 may be affected with associated variables of: $Q_{13}^{12}, Q_{23}^{12}$, and $Q_{23}^{22}$.

The remainder of the data is as in Example 5.

The computed equilibrium solution for this example is:

$$
\begin{gathered}
Q_{11}^{11 *}=0.00, \quad Q_{21}^{11 *}=0.00, \quad Q_{12}^{11 *}=23.74, \quad Q_{22}^{11 *}=66.26, \\
Q_{13}^{12 *}=76.26, \quad Q_{23}^{12 *}=83.74, \quad Q_{23}^{22 *}=140.00, \\
\lambda_{1}^{1 *}=1904.02, \quad \lambda_{2}^{1 *}=1666.70, \quad \lambda_{2}^{2 *}=682.28,
\end{gathered}
$$

and $\gamma_{3}^{*}=366.21$.

Observe that all the Lagrange multipliers are positive and, therefore, on the supply side all of the convalescent plasma available is donated and on the demand side, the for profit collection site is at its capacity for handling the plasma donated there.

We see that, as compared to the results in Example 5, now there is a positive amount of convalescent plasma donated by class 1 and origin location 2 to nonprofit collection site 1 and an increase in convalescent plasma donations from this class and location to collection site 2 . However, because of the capacity on collection site 3 , the volume of plasma donations there decreases from donors of class 1 . Class 2 , which is unable to donate for immediate transfusion purposes, again, donates all of its plasma to the for profit site 3 .

Some of the insights gained from the above numerical examples, although stylized, are:

1. It is important to make the experience of donating convalescent plasma as positive as feasible since a decrease in a utility function fixed term can impact donations.

2. Care should be taken when a for profit moves in since convalescent plasma donors may shift their donations from nonprofit organizations to a for profit one.

3. Proximity matters and convenience of collection sites.

4. Availability of labor needed for the collection process during the pandemic and capacities of the collection sites play an important role. Organizations collecting convalescent plasma need to have the resources to collect from the donors.

\section{Summary and Conclusions and Suggestions for Future Research}

In this paper, we developed a multiclass, multiproduct equilibrium model to study the behavior of convalescent plasma donors in the Covid-19 pandemic. As more and more countries across the globe approve clinical trials for convalescent plasma therapy to fight the Covid-19 pandemic and as collections of this 
life-saving product gain momentum, it will be critical to understand how different classes of donors located at different sites choose to donate to different organizations. Antibody-rich plasma from recovered Covid-19 patients has not only been used in severely ill patients, but also to develop other products such as hyperimmune globulin. Each location in our model has a fixed population of convalescent plasma donors who meet the eligibility criteria. The donor population can be separated into several classes based on their motivation to donate and the product for which they are donating at a specific location. Preferences of each class of donor at an origin location for donating for a convalescent plasma product at a collection site is captured in the utility functions. While people make charitable donations driven by altruism, there are tangible factors associated with the effort to donate that might deter donors. It requires time and cost to travel to a plasma collection site to provide donations. Often donors have to wait for their turn and the collection process itself takes about an hour. Moreover, during a pandemic there are risks of infection associated with traveling, specially if the collection sites are far from the donor's location as well as the possibility of an accident. All these important factors affecting donor decisions are captured in our generalized cost functions.

To the best of our knowledge this is the first model to capture competition for convalescent plasma between nonprofit organizations such as blood banks and hospitals, and for-profit organizations such as pharmaceutical companies that are working on the development of plasma derived products. An extension of the convalescent plasma model is provided to include capacity restrictions at collection sites. We provide equilibrium conditions for both capacitated and uncapacitated models. Qualitative properties of the model and the algorithmic framework of modified projection method are also outlined. The algorithm is implemented and applied to solve a series of numerical examples that explore several scenarios with varying levels of complexity. The results obtained from these numerical examples reveal how nonprofit organizations might lose donations due to competition from for-profit organizations that provide monetary compensations to donors. The insights obtained from this analysis can help policy makers understand the behavior pattern of convalescent plasma donors under different circumstances.

As the studies on convalescent plasma therapy make progress and more data are accessible on the availability and collection of convalescent plasma, our model can be further tested using real data. Another possible extension of this work would involve capturing the stochastic nature of the supply and demand for convalescent plasma. Furthermore, since there is the possibility of repeat donations of convalescent plasma, adding that feature to the model would be interesting. Constructing a game theory model with objective functions for the nonprofit and the for-profit organizations competing for a limited amount of convalescent plasma would also be worthwhile. Furthermore, it is important to emphasize that the model can also inform in future disease outbreaks and pandemics, in which convalescent plasma may play a role in medical treatments.

Every global tragedy in human history dating back to World War II has paved the way for scientific innovation, technological advancement, and better understanding of the psyche of society at large. This Covid-19 pandemic is not any different. 
The Operations Research and Management Science community has the skill set and mathematical and computational tools needed to analyze the evolving situation with the outbreak of the disease. Research on the pandemic using Operations Research and Management Science tools is important and can help inform and guide decision makers also in the area of public policy. With this paper we contribute to the literature with the development of a new model for convalescent plasma in a unique market that has arisen in the Covid-19 pandemic. The model handles different classes of donors, as well as different convalescent plasma products, and allows for the investigation, in its computational form, a multiplicity of scenarios such as the impact of the addition of classes, the addition of donor origin locations, and the addition of nonprofit collection sites and a for profit competitor collection site. Of course, changes to the underlying functions is also made possible and we illustrated this as well in the form of changes in utility functions.

Acknowledgements The authors thank the two anonymous reviewers, the Associate Editor and the Editor in Chief for helpful comments and suggestions on two earlier versions of this paper. This paper is dedicated to essential workers, including the excellent systems administrators in Engineering Computer Services at UMass Amherst, who even in a pandemic assisted the first author, notably, Jose Figueroa and Stephen Cumberbatch, and to all the tech workers, healthcare workers, first responders, grocery store workers, and freight service providers, whose dedication and extraordinary efforts during the Covid-19 pandemic helped to sustain us. Thank you. We also recognize all those who have suffered from Covid-19 and memorialize those who perished from this disease.

Funding Information No funding was provided for this work.

Data Availability Statement All data generated or analysed during this study are included in this paper.

\section{Declarations}

Conflict of Interest The authors declare that they have no conflict of interest

\section{References}

1. Holder J (2021) Tracking coronavirus vaccinations around the world. The New York Times, May 21. https://www.nytimes.com/interactive/2021/world/covid-vaccinations-tracker.html

2. Magalhaes L, Pearson S (2021) Brazil Covid-19 variant tears through South America in warning to world. The World Street Journal, April 28. https://www.wsj.com/articles/brazil-covid-19-variantspreads-across-south-america-in-warning-to-world-11619611204

3. Reuters (2021) UK daily COVID cases highest in a month, Indian variant rising sharply. Reuters, May 20. https://www.reuters.com/world/uk/uk-records-2874-covid-19-cases-highest-daily-total-month-2021-05-20/

4. Pandey V, Nazmi S (2021) Covid-19 in India: Why second coronavirus wave is devastating. BBC News, April 21 https://www.bbc.com/news/world-asia-india-56811315

5. Bloch EM, Shoham S, Casadevall A, Sachais BS, Shaz B, Winters JL, van Buskirk C, Grossman BJ, Joyner M, Henderson JP, Pekosz A (2020) Deployment of convalescent plasma for the prevention and treatment of COVID-19. J Clin Inv, in press

6. Casadevall A, Pirofski LA (2020) The convalescent sera option for containing COVID-19. J Clin Investig 130(4):1545-1548

7. Duan K, Liu B, Li C, Zhang H, Yu T, Qu J, Zhou M, Chen L, Meng S, Hu Y, Peng C (2020) Effectiveness of convalescent plasma therapy in severe COVID-19 patients. Proc Natl Acad Sci 117(17):9490-9496 
8. Shen C, Wang Z, Zhao F, Yang Y, Li J, Yuan J, Wang F, Li D, Yang M, Xing L, Wei J (2020) Treatment of 5 critically ill patients with COVID-19 with convalescent plasma. JAMA 323(16):1582-1589

9. Hererra T (2020) What is convalescent blood plasma, and why do we care about it? The New York Times, April 24. https://www.nytimes.com/2020/04/24/smarter-living/coronavirus-convalescentplasma-antibodies.html

10. Beasley D, (2020) Donated plasma benefits COVID-19 patients in small U.S. study. Reuters, May 22. https://www.reuters.com/article/us-health-coronavirus-plasma/donated-plasma-benefits-covid19-patients-in-small-u-s-study-idUSKBN22Y303

11. Fabricius MM, Dandachi D (2021) COVID-19 convalescent plasma: From donation to treatment-A systematic review and single Center experience. Mo Med 118(1):74

12. Shaz B, Dunbar C, Hillyer C, Hari P, Gernsheimer T, Davey R, Bloch E (2021) COVID-19 and convalescent plasma and antibody therapies: Frequently asked questions. American Society of Hematology, updated March 23. https://www.hematology.org/covid-19/covid-19-and-convalescent-plasma

13. Harris R (2021) Convalescent plasma strikes out as COVID-19 treatment. NPR, March 10. https:// www.npr.org/sections/health-shots/2021/03/10/975365309/convalescent-plasma-strikes-out-ascovid-19-treatment

14. Kaunain MS (2021) India drops plasma therapy from Covid treatment protocol. The Indian Express, May 18. https://indianexpress.com/article/india/india-drops-plasma-therapy-covid-treatment-7319290/

15. Ferguson C, McFadden C, Martinez D, Schapiro R, (2020) Convalescent plasma is safe to treat COVID-19: nationwide study. NBC News, May 14. https:/www.nbcnews.com/health/health-news/ convalescent-plasma-safe-treat-covid-19-nationwide-study-n1206126

16. Cheng Y, Wong R, Soo YOY, Wong WS, Lee CK, Ng MHL, Chan P, Wong KC, Leung CB, Cheng $\mathrm{G}$ (2005) Use of convalescent plasma therapy in SARS patients in Hong Kong. Eur J Clin Microbiol Infect Dis 24(1):44-46

17. Winkler AM, Koepsell SA (2015) The use of convalescent plasma to treat emerging infectious diseases: focus on Ebola virus disease. Curr Opin Hematol 22(6):521-526

18. Van Griensven J, Edwards T, de Lamballerie X, Semple MG, Gallian P, Baize S, Horby PW, Raoul H, Magassouba NF, Antierens A, Lomas C (2016) Evaluation of convalescent plasma for Ebola virus disease in Guinea. N Engl J Med 374(1):33-42

19. Mair-Jenkins J, Saavedra-Campos M, Baillie JK, Cleary P, Khaw FM, Lim WS, Makki S, Rooney K.D, Convalescent Plasma Study Group, Nguyen-Van-Tam JS, Beck CR (2015) The effectiveness of convalescent plasma and hyperimmune immunoglobulin for the treatment of severe acute respiratory infections of viral etiology: a systematic review and exploratory meta-analysis. J Infect Dis 211(1):80-90

20. European Blood Alliance (2020) Convalescent plasma (CCP). https://europeanbloodalliance.eu/ activities/convalescent-plasma-cpp/

21. Fortuna G (2021) COVID-19 plasma therapy comes handy either way, EU Commission says. EURACTIV, April 1. https://www.euractiv.com/section/health-consumers/news/covid-19-plasmatherapy-comes-handy-either-way-eu-commission-says/

22. MD Bureau (2020) ICMR Gets National Ethics Committee Nod For COVID-19 Plasma Therapy. Medical Dialogues, May 9. https://medicaldialogues.in/news/health/government-policies/icmr-getsnational-ethics-committee-nod-for-covid-19-plasma-therapy-65599

23. WION (2021) COVID-19 Donating plasma: Here's everything you need to know. WION, May 13. https:// www.wionews.com/india-news/covid-19-donating-plasma-heres-everything-you-need-to-know-384733

24. Sheridan C (2020) Convalescent serum lines up as first-choice treatment for coronavirus. May 7 , Nature Biology. https://www.nature.com/articles/d41587-020-00011-1

25. Aleccia J (2020) Market for blood plasma from COVID-19 survivors heats up. NPR, May 11. https://www.npr.org/sections/health-shots/2020/05/11/852354920/market-for-blood-plasma-fromcovid-19-survivors-heats-up

26. Bloch EM (2021) COVID-19: Convalescent plasma and hyperimmune globulin. UptoDate, March 10. https://www.uptodate.com/contents/covid-19-convalescent-plasma-and-hyperimmune-globulin

27. Food and Drug Administration (2020a) COVID-19 convalescent plasma collection: Donor eligibility, processing, labeling, and distribution. April 4. https:/www.fda.gov/vaccines-blood-biologics/ investigational-new-drug-ind-or-device-exemption-ide-process-cber/recommendations-investigationalcovid-19-convalescent-plasma 
28. Davies E (2020) As states begin to reopen, blood banks brace for surge in demand. The Washington Post, May 22. https://www.washingtonpost.com/local/as-states-begin-to-reopen-blood-banksbrace-for-surge-in-demand/2020/05/22/0dbd25ec-9add-11ea-89fd-28fb313d1886_story.html

29. Food and Drug Administration (2020b) Coronavirus (COVID-19) update. December 1. https:// www.fda.gov/news-events/press-announcements/coronavirus-covid-19-update-december-1-2020

30. City News Service (2020) San Diego Blood Bank asks for convalescent plasma as COVID-19 cases increase. KPBS, December 2. https://www.kpbs.org/news/2020/dec/02/san-diego-blood-bank-asksconvalescent-plasma-covi/

31. Tracy K (2020) 1 donation could save 5 lives I COVID-19 convalescent plasma donations in high demand as cases surge. First Coast News, December 7. https://www.firstcoastnews.com/article/ news/health/coronavirus/covid-19-convalescent-plasma-donations-needed-one-donation-couldsave-five-lives/77-45d569e8-ce0f-493d-b826-8c096a7e1138

32. WBIR Staff (2020) MEDIC expands COVID-19 convalescent plasma donation appointments. 10News, December 1. https:/www.wbir.com/article/news/health/medic-expands-covid-19-convalescent-plasmadonation-appointments/51-d690940d-053d-4711-a7af-b1389341be67

33. Aleccia J, (2021) Convalescent plasma hype has faded but some say the Covid therapy still holds promise. NBCNews, March 21. https://www.nbcnews.com/health/health-news/convalescent-plasmahype-has-faded-some-say-covid-therapy-still-n1261567

34. Food and Drug Administration (2020c) Investigational COVID-19 convalescent plasma. February 11. https://www.fda.gov/media/136798/download

35. Gontu A, Srinivasan S, Salazar E, Nair MS, Nissly RH, Greenawalt D, Bird IM, Herzog CM, Ferrari MJ, Poojary I, Katani R (2021) Limited window for donation of convalescent plasma with high livevirus neutralizing antibody titers for COVID-19 immunotherapy. Commun Biol 4(1):1-9

36. American Red Cross (2020) FAQ: COVID-19 convalescent plasma. https://www.redcrossblood.org/ faq.html\#donating-blood-covid-19-convalescent-plasma

37. Bradley J (2020) Blood samples, vital for antibody tests, sold at exorbitant rates. May 1, The New York Times. https://www.nytimes.com/2020/05/01/world/europe/coronavirus-blood-samples.html

38. Life Serve Blood Center (2020) Questions about convalescent plasma. https://www.lifeservebloodc enter.org/donate/recovered-covid-19-patient-plasma-donation/questions-convalescent-plasma/

39. Korcok M (2002) Blood donations dwindle in US after post-Sept. 11 wastage publicized. CMAJ: Canadian Medical Association Journal 167(8), 907

40. Andreoni J (1990) Impure altruism and donations to public goods: A theory of warm-glow giving. Econ J 10(401):464-477

41. Gillespie TW, Hillyer CD (2002) Blood donors and factors impacting the blood donation decision. Transfus Med Rev 16(2):115-130

42. Evans R, Ferguson E (2013) Defining and measuring blood donor altruism: A theoretical approach from biology, economics and psychology. Vox Sang 106(2):118-26

43. Craig AC, Garbarino E, Heger SA, Slonim E (2017) Waiting to give: Stated and revealed preferences. Manage Sci 63(11):3672-3690

44. Nagurney A, Dutta P (2019) Supply chain network competition among blood service organizations: a Generalized Nash Equilibrium framework. Ann Oper Res 275(2):551-586

45. Farrugia A, MacPherson J, Busch MP (2020) Convalescent plasma this is no time for competition. Transfusion, June 25. https://www.ncbi.nlm.nih.gov/pmc/articles/PMC7323360/

46. Toyasaki F, Wakolbinger T (2014) Impacts of earmarked private donations for disaster fundraising. Ann Oper Res 221(1):427-447

47. Saxton GD, Zhuang J (2013) A game-theoretic model of disclosure-donation interactions in the market for charitable contributions. J Appl Commun Res 41(1):40-63

48. Zhuang J, Saxton GD, Wu H (2014) Publicity vs. impact in nonprofit disclosures and donor preferences: A sequential game with one nonprofit organization and $\mathrm{N}$ donors. Ann Oper Res 221(1):469-491

49. Kessler JB, Roth AE (2012) Organ allocation policy and the decision to donate. Am Econ Rev 102(5):2018-2047

50. Kinderlehrer D, Stampacchia G (1980) An Introduction to Variational Inequalities and Their Applications. Academic Press, New York

51. Dafermos S (1980) Traffic equilibrium and variational inequalities. Transp Sci 14:42-54

52. Dafermos S (1982) The general multimodal network equilibrium problem with elastic demand. Networks 12:57-72 
53. Gabay D, Moulin H (1980) On the uniqueness and stability of Nash equilibria in noncooperative games. In: Bensoussan A, Kleindorfer P, Tapiero CS (eds) Applied Stochastic Control of Econometrics and Management Science. North-Holland, The Netherlands, pp 271-294

54. Florian M, Los M (1982) A new look at static spatial price equilibrium models. Reg Sci Urban Econ 12:579-597

55. Daniele P, Giannessi F, Maugeri A (eds) (2003) Equilibrium Problems and Variational Models. Kluwer Academic Publishers, Norwell, Massachusetts

56. Pardalos PM, Migdalas A, Pitsoulis L (eds) (2008) Pareto Optimality, Game Theory and Equilibria. Springer-Verlag, New York

57. Daniele P, Scrimali L, Editors, (2018) New Trends In Emerging Complex Real Life Problems. Springer Nature Switzerland AG

58. Battiato S, Daniele P, Farinella GM, Giuffre S, Scrimali LRM (2018) Preface. J Global Optim 70:307

59. Muggy L, Stamm JLH (2014) Game theory applications in humanitarian operations: A review. J Human Logis Supply Chain Manag 4(1):4-23

60. Nagurney A (1989) Migration equilibrium and variational inequalities. Econ Lett 31:109-112

61. Nagurney A (1990) A network model of migration equilibrium with movement costs. Math Comput Model 13:79-88

62. Nagurney A, Pan J, Zhao L (1992) Human migration networks. Eur J Oper Res 59:262-274

63. Nagurney A,PanJ,ZhaoL(1992)Human migrationnetworkswithclasstransformations.In:Lakshmanan TR, Nijkamp P (eds) Structure and Change in the Space Economy. Springer-Verlag, Berlin, Germany, pp 239-258

64. Causa A, Jadamba B, Raciti F (2017) A migration equilibrium model with uncertain data and movement costs. Decis Econ Finance 40(1): 159-175

65. Nagurney A, Daniele P (2020) International human migration networks under regulations. Euro J Oper Res, in press

66. Nagurney A (1999) Network Economics: A Variational Inequality Approach, second and, revised. Kluwer Academic Publishers, Dordrecht, The Netherlands

67. Dutta P, Nagurney A (2019) Multitiered blood supply chain network competition: Linking blood service organizations, hospitals, and payers. Oper Res Health Care 23:100230

68. Piraban A, Guerrero WJ, Labadie N (2019) Survey on blood supply chain management: Models and methods. Comput Oper Res 112:104756

69. Osorio AF, Brailsford SC, Smith HK, Forero-Matiz SP, Camacho-Rodriguez BA (2017) Simulation-optimization model for production planning in the blood supply chain. Health Care Manag Sci 20(4):548-564

70. Masoumi AH, Yu M, Nagurney A (2017) Mergers and acquisitions in blood banking systems: A supply chain network approach. Int J Prod Econ 193:406-421

71. Fazli-Khalaf M, Khalilpourazari S, Mohammadi M (2019) Mixed robust possibilistic flexible chance constraint optimization model for emergency blood supply chain network design. Ann Oper Res 283:1079-1109

72. Khalilpourazari S, Khamseh AA (2019) Bi-objective emergency blood supply chain network design in earthquake considering earthquake magnitude: a comprehensive study with real world application. Ann Oper Res 283:355-393

73. Samani MRG, Torabi SA, Hosseini-Motlagh SM (2018) Integrated blood supply chain planning for disaster relief. Int J Dis Risk Red 27:168-188

74. Salehi F, Mahootchi M, Husseini SMM (2019) Developing a robust stochastic model for designing a blood supply chain network in a crisis: A possible earthquake in Tehran. Ann Oper Res 283(1-2):679-703

75. Hosseini-Motlagh SM, Samani MRG, Cheraghi S (2020) Robust and stable flexible blood supply chain network design under motivational initiatives. Socioecon Plann Sci 70:100725

76. Ferguson E, France CR, Abraham C, Ditto B, Sheeran P (2007) Improving blood donor recruitment and retention: integrating theoretical advances from social and behavioral science research agendas. Transfusion 47(11):1999-2010.

77. Schlumpf KS, Glynn SA, Schreiber GB, Wright DJ, Randolph Steele W, Tu Y, Hermansen S, Higgins MJ, Garratty G, Murphy E (2008) Factors influencing donor return. Transfusion 48(2):264-272

78. Schreiber GB, Schlumpf KS, Glynn SA, Wright DJ, Tu YL, King MR, Higgins MJ, Kessler D, Gilcher R, Nass CC, Guiltinan AM (2006) Convenience, the bane of our existence, and other barriers to donating. Transfusion 46:545-553 
79. Lacetera N, Macis M, Slonim R (2013) Economic rewards to motivate blood donations. Science 340(6135):927-928

80. Kominers SD, Pathak PA, Snmez T, Denver MU (2020) Paying it backward and forward: Expanding access to convalescent plasma therapy through market design. National Bureau of Economic Research, No. w27143

81. Kabir KA, Tanimoto J (2020) Evolutionary game theory modelling to represent the behavioural dynamics of economic shutdowns and shield immunity in the COVID-19 pandemic. Royal Soc Open Sci 7(9):201095

82. Kaplan EH (2020) Containing 2019-nCoV (Wuhan) coronavirus. Health Care Management Science, 1-4. https://doi.org/10.1007/s10729-020-09504-6

83. Baveja A, Kapoor A, Melamed B, (2020) Stopping Covid-19: A pandemic-management service value chain approach. Ann Oper Res, in press. https://doi.org/10.1007/s10479-020-03635-3

84. Ivanov D (2020) Viable supply chain model: integrating agility, resilience and sustainabil- ity perspectivesllessons from and thinking beyond the COVID-19 pandemic. Ann Oper Res. https://doi. org/10.1007/s10479-020-03640-6

85. Rassia ST (2020) How architecture fails in conditions of crisis: A discussion on the value of interior design over the COVID-19 outbreak. SN Operations Research Forum 1, 13. https://doi.org/10.1007/ s43069-020-0014-9

86. Nagurney A (2021) Supply chain game theory network modeling under labor constraints: Applications to the Covid-19 pandemic. Eur J Oper Res 293(3):880-891

87. Choi TM (2021) Fighting against COVID-19: what operations research can help and the sense-andrespond framework. Annals Oper Res, in press. https://doi.org/10.1007/s10479-021-03973-w

88. New York Blood Center (2020) Convalescent plasma- donor FAQ. https://nybloodcenter.org/donateblood/covid-19-and-blood-donation-copy/convalescent-plasma-donor-faq/

89. Sundermann LM (2018) Share experiences: receiving word of mouth and its effect on relationships with donors. J Serv Mark 32(3):322-333

90. Martin S, Greiling D, Leibetseder N (2019) Effects of word-of-mouth on the behavior of Austrian blood donors: a case study of the Red Cross Blood Donation Service. Health Promot Int 34(3):429-439

91. Williams M, Buttle F (2013) Managing word-of-mouth: A nonprofit case study. J Nonprofit Public Sect Mark 25(3):284-308

92. Korpelevich GM (1977) The extragradient method for finding saddle points and other problems. Matekon 13:35-49

93. Nagurney A, Salarpour M, Nagurney LS (2020) A stochastic disaster relief game theory network model. SN Oper Res Forum 1:10

94. Nagurney A, Zhang D (1996) Projected Dynamical Systems and Variational Inequalities with Applications. Kluwer Academic Publishers, Boston, Massachusetts

95. Pawlowski A (2020) What is plasma? How to donate convalescent plasma for coronavirus patients. Today, April 23. https://www.today.com/health/what-plasma-donating-convalescent-covid-19plasma-coronavirus-patients-t179700

96. Chadha J (2020) New York City's most crowded neighborhoods are often hardest hit by coronavirus. Politico, April 11. https://www.politico.com/states/new-york/albany/story/2020/04/11/newyork-citys-most-crowded-neighborhoods-are-often-hardest-hit-by-coronavirus- 1274875

Publisher's Note Springer Nature remains neutral with regard to jurisdictional claims in published maps and institutional affiliations. 